\section{Editorial comments: treatment of motor fluctuations in Parkinson's disease}

\author{
Amos D. Korczyn
}

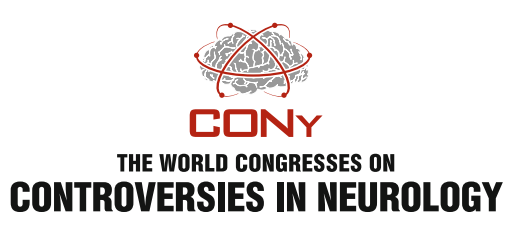

Published online: 27 May 2011

(C) The Author(s) 2011. This article is published with open access at Springerlink.com

This timely debate tries to address a common clinical problem faced by movement disorders specialists. Following the initial impressive improvement gained by dopaminergic medications, many patients develop dyskinesias and fluctuations which are clearly drug related. The pathogenesis of these problems is still unclear, but the most reasonable explanation is that with the ongoing degeneration of dopaminergic pathways in the striatum, the ability of these terminals to regulate or stabilize the synaptic concentration of dopamine is lost. Thus a direct relationship between blood levels and motor effect is reached, leading to insufficient or excessive stimulation of postsynaptic dopamine receptors (Korczyn 1972). Attempts to control the dopaminergic tone by frequent smaller doses are of minor help at best.

These pharmacokinetic considerations have led to the development of two methods which allow continuous stable serum levels of dopamine agonists, the apomorphine pump which delivers apomorphine subcutaneously, and the intraduodenal delivery of levodopa (with carbidopa) through a percutaneous enteric gastrostomy (PEG). Both systems have now been used for a number of years and provide significant benefit.

Intracranial surgical attempts to control parkinsonian symptoms are much older than these approaches. Ablative surgery at various sites can control tremor, while newer approaches have been developed which can also affect other motor symptoms. The newest target is the subthalamic nucleus, and rather than destructive lesions the

A. D. Korczyn $(\bowtie)$

Tel Aviv, Israel

e-mail: amoskor@post.tau.ac.il modern approach is to stimulate this area. Presumably the electrical stimulation is inhibitory, although the exact neural circuitry which is affected and the mechanism of activity are still far from being fully understood.

Early studies in University Hospital, Grenoble, France, have succeeded to overcome the initial reluctance of neurologists to submit their patients to surgery. Over the past two decades, several centers over the world have been able to confirm these results.

As both papers mention, there are no head-to-head studies which compare these methods, and it is perhaps not surprising that each center maintains the method in which it gained experience.

The specialist facing a patient with motor fluctuations must, therefore, assume that these methods are comparable in their outcome, and thus the decision must be made individually in each case. The indications are similar, but there are differences in contraindications.

As both papers stress, the final decision as to the choice of method must rest with the patient. However, the critical recommendation of the specialist must depend on the experience of the teams in the geographical area.In that respect, DBS is significantly more demanding. It requires a dedicated team of neurologists, neurosurgeons, radiologists, psychologists and neurophysiologists to evaluate the patient and perform the surgery. Moreover, the post-operative care may demand adjustment of the current parameter which is usually done by the neurologist. Thus it is clear that only centers with a dedicated team should be allowed to perform the surgical intervention.

The Grenoble team depends on electrophysiological identification of the stimulation target. This time and effort consuming procedure is not performed in several other centers, who claim to have equally good results. 
As stressed by Carron et al., the pre-surgical discussion must clarify to the patient what are the reasonable expectations. The main aim of surgery should be to prevent motor fluctuations and reduce dyskinesias. Non-motor fluctuations and freezing do not respond well. The patient should not assume that he will be "cured" of PD, and be warned that the disease will continue to develop, leading to additional non-motor disturbances which will seriously affect the quality of life. There is no evidence that any of the methods discussed are neuroprotective or affect the rate of progression.

The three approaches are very costly, and this factor needs to be taken into consideration when selecting the intervention.
Open Access This article is distributed under the terms of the Creative Commons Attribution Noncommercial License which permits any noncommercial use, distribution, and reproduction in any medium, provided the original author(s) and source are credited.

\section{Reference}

Korczyn MD (1972) Pathophysiology of drug-induced dyskinesias. Neuropharmacol 11:601-607 